\title{
Cardiovascular disease and intensive glucose control in type 2 diabetes mellitus: moving practice toward evidence-based strategies
}

REVIEW

This article was published in the following Dove Press journal:

Vascular Health and Risk Management

16 October 2009

Number of times this article has been viewed

\author{
Matthias Meier ${ }^{1,2}$ \\ Michael Hummel ${ }^{3,4}$ \\ 'Clinic for Hypertension and \\ Nephrology, Hannover, Germany; \\ ${ }^{2}$ Department of Nephrology, \\ Hannover Medical School, Hannover, \\ Germany; ${ }^{3}$ Academic Hospital \\ Schwabing, Munich, Germany; \\ ${ }^{4}$ Diabetes Research Institute, Munich, \\ Germany
}

\begin{abstract}
Type 2 diabetes mellitus (T2DM) is associated with a high risk of complications, essentially macrovascular events. Surprisingly, the effect of improved glucose control on coronary and cerebrovascular complications and the target level of glycated hemoglobin $\left(\mathrm{HbA}_{1 \mathrm{c}}\right)$ in this population remains questionable. We here report the results of 4 recently published randomized controlled trials (ACCORD, ADVANCE, VADT, UKPDS post-trial), which did not demonstrate a significant reduction of cardiovascular events in the intensive group compared to the standard group. On the contrary, in ACCORD, the study with the most ambitious goal $\left(\mathrm{HbA}_{1 \mathrm{c}}<6 \%\right.$ ), the overall and cardiovascular mortality was greater in the intensive group, although the risk of microangiopathic complications, especially nephropathy, was significantly decreased. VADT suggests that one possibility for the lack of observed effect of intensive therapy could be that the cardiovascular benefit is delayed. This contrasts strongly with the long-term postintervention outcomes of UKPDS, which show a persistent benefit of glycemic control during 10 years of post-trial follow-up ('legacy effect'). Therefore, the best way to protect patients with T2DM against coronary and cerebrovascular disease is to target all cardiovascular risk factors as early as possible by an individualized approach.
\end{abstract}

Keywords: glycemic control, cardiovascular, ACCORD, ADVANCE, VADT, UKPDS post-trial

\section{Introduction}

The macro- and microvascular burden of type 2 diabetes mellitus (T2DM) is well established. Mortality from cardiovascular disease (CVD) is increased by a factor of 2 to 3 in persons with diabetes mellitus compared with the general population. ${ }^{1} \mathrm{CVD}$ develops earlier in the presence of diabetes mellitus and occurs as often in diabetic women as in diabetic men. ${ }^{1}$ A number of recent single risk factor intervention trials targeting hyperglycemia, hypertension, dyslipidemia, procoagulation, microalbuminuria, and existing cardiovascular disorders have shown major beneficial effects on long-term outcome. ${ }^{2}$ However, the Steno- 2 study with an intensive intervention for an average of 7.8 years, showed an event rate of the combined cardiovascular endpoint of $7 \%$ per year. ${ }^{3,4}$ Although the intensified multifactorial intervention cuts cardiovascular events as well as nephropathy, retinopathy, and autonomic neuropathy by about half, it is still more than three times as high as in the matched background population, leaving much room for more improvements. ${ }^{3,4}$ To further reduce this increased risk, a multifactorial approach to the management of T2DM has now been advocated, and most of the updated national guidelines for the treatment of T2DM recommend a versatile approach driven by ambitious treatment targets. The American Diabetes Association (ADA), for example, recommends not only good glycemic control but
Correspondence: Matthias Meier

Clinic for Hypertension and Nephrology, Heidering 3I, 30625 Hannover, Germany Tel +495II 542630

Fax +495II 5426310

Email kfnh_hannover@web.de 
also identification and aggressive treatment of associated cardiovascular risk factors, with more stringent target levels for lipids and blood pressure (BP) than those recommended for the general population. ${ }^{5}$

\section{Chronic hyperglycemia and cardiovascular disease: cause or link?}

Though there is a link between hyperglycemia and cardiovascular risk, there is less evidence that glucose lowering is associated with reduction in CVD risk. Patients with T2DM whose glycated hemoglobin $\left(\mathrm{HbA}_{1 \mathrm{c}}\right)$ levels were reduced from $8 \%$ to $7 \%$ in the United Kingdom Prospective Diabetes Study (UKPDS) did not exhibit a reduction in CVD, although a subgroup of patients treated with metformin had a lower risk of cardiovascular events. ${ }^{6}$ Among patients with type 1 diabetes mellitus (T1DM) studied in the Diabetes Control and Complications Trial/Epidemiology of Diabetes Interventions and Complications trial (DCCT/EDIC), glucose lowering was associated with a long-term benefit with regard to cardiovascular complications that became apparent only years after recruitment. ${ }^{7,8}$

Four recent studies, the ACCORD (Action to Control Cardiovascular Risk in Diabetes) study, ${ }^{9}$ the ADVANCE (Action in Diabetes and Vascular Disease: Preterax and Diamicron Modified Release Controlled Evaluation) trial, ${ }^{10}$ the VADT (Veterans Affairs Diabetes Trial) ${ }^{11}$ trial and the United Kingdom Prospective Diabetes Study Post-Trial (UKPDS post-trial) study, ${ }^{12}$ sought to determine the effect of lowering glucose to near-normal levels on cardiovascular risk. All studies share a number of common features, and were well designed and randomized. Each study was designed to evaluate the effects of intensive treatment for glycemic control in comparison to standard glucose-lowering targets on vascular outcomes in patients with T2DM who were considered to be at high risk. ${ }^{13}$ All studies evaluated an intensive blood-glucose-control strategy, rather than a specific therapeutic regimen, to achieve glycemic targets at levels well below those that are currently recommended. However, the trials differed substantially as displayed in Table 1. Thus, what did these studies show in more detail?

\section{ACCORD}

The ACCORD trial, a multicenter study of T2DM sponsored by the National Institutes of Health (NIH), was designed primarily to examine the effects of strict glycemic control on CVD in subjects with long-standing diabetes mellitus. ${ }^{5,9}$ The study cohort of 10,250 adults (mean age 62 years) with a median diabetes duration of 10 years (including 35\% already treated with insulin at baseline) and at high risk for CVD (diagnosed with CVD or 2 risk factors in addition to diabetes mellitus) was randomly assigned to an intensive treatment group with the aim of achieving $\mathrm{HbA}_{1 \mathrm{c}}$ of $<6 \%$ or a standard treatment group with an $\mathrm{HbA}_{1 \mathrm{c}}$ goal of $7.0 \%$ to $7.9 \%{ }^{5,9}$ The diabetes treatment strategies took advantage of an algorithmic approach using numerous diabetes medications in both study groups. ${ }^{5,9}$ In addition, in this factorial study, approximately one-half of the population was assigned to a BP study (intensive therapy aiming for systolic BP $<120$ and conventional aiming for systolic $\mathrm{BP}<140 \mathrm{mmHg}$ ) and one-half to a lipid study in which all subjects were treated with a statin, but one-half randomly assigned to fenofibrate and one-half to placebo (see below). ${ }^{5,9}$

From a baseline median $\mathrm{HbA}_{1 \mathrm{c}}$ of $8.1 \%$, the intensive arm reached a median $\mathrm{HbA}_{1 \mathrm{c}}$ of $6.4 \%$ compared to $7.5 \%$ in the control arm within 12 months of randomization. ${ }^{5,9}$ Compared to standard group, the use of insulin in combination with multiple oral agents, weight gain, and episodes of severe hypoglycemia was increased significantly more in the intensive treatment group. 5,9

In 2008, the intensive blood sugar lowering arm of the study was halted based upon a recommendation of the external Data Safety Monitoring Board due to a higher number of total and cardiovascular deaths (257 vs 203) in subjects assigned to intensive therapy (HR 1.22; 95\% CI 1.01 to 1.46$).{ }^{5,9}$ However, the embedded BP and lipid studies are currently ongoing. Over an average of 3.5 years, there was an excess of three deaths per 1000 subjects per year in the intensive group. Notably, the rate of death in both treatment groups was lower than that reported in other studies of T2DM. The primary outcome (a composite of nonfatal myocardial infarction (MI), nonfatal stroke, or death from cardiovascular causes) occurred in 352 and 371 patients in the intensive and standard therapy groups, although this finding was not statistically significant (0.90 [0.78 to 1.04$], P=0.16){ }^{5,9}$

Extensive analyses have not identified a specific cause for the excess mortality. ${ }^{5,9}$ Subjects in the intensive group rapidly achieved target $\mathrm{HbA}_{1 \mathrm{c}}$ values (median $\mathrm{HbA}_{1 \mathrm{c}}$ decreased from $8.1 \%$ to $6.7 \%$ in 4 months and was $6.4 \%$ after 3.5 years) and experienced a greater number of severe hypoglycemic events (annualized rate of 3.1 vs $1.0 \%$ ) and more weight gain (mean $3.5 \mathrm{vs} 0.4 \mathrm{~kg}$ at 3 years) than the standard group. ${ }^{5,9}$ However, more frequent hypoglycemia did not apparently account for the difference in the death rate, nor did a specific medication or combination of medicines. ${ }^{5,9}$ The design of ACCORD limits the ability to determine whether the differences in 
Table I Comparison of four trials of intensive glycemic control and CVD outcomes

\begin{tabular}{|c|c|c|c|c|}
\hline & ACCORD & ADVANCE & VADT & UKPDS post-trial \\
\hline $\begin{array}{l}\text { Clinical trials } \\
\text { NIH listing }\end{array}$ & $\begin{array}{l}\text { NCT00000620 } \\
\text { see website } \\
\text { http://clinicaltrials.gov }\end{array}$ & $\begin{array}{l}\text { NCT00I45925 } \\
\text { see website } \\
\text { http://clinicaltrials.gov }\end{array}$ & $\begin{array}{l}\text { NCT00032487 } \\
\text { see website } \\
\text { http://clinicaltrials.gov }\end{array}$ & $\begin{array}{l}\text { ISRCTN7545I837 } \\
\text { see website } \\
\text { http://controlled-trials.com }\end{array}$ \\
\hline Design & RCT, AC, Phase III & RCT, PC, Phase III & RCT, AC, Phase III & RCT, AC, Phase III \\
\hline \multicolumn{5}{|l|}{ Participant characteristics } \\
\hline$n$ & $|0,25|$ & $\mathrm{II}, \mathrm{I} 40$ & $|, 79|$ & 3,277 \\
\hline Mean age (years) & 62 & 66 & 60 & 63 \\
\hline Duration of diabetes (years) & 10 & 8 & 11.5 & 17 \\
\hline Sex (\% male/female) & $39 / 61$ & $42 / 58$ & $97 / 3$ & SI:60/40; M:46/54 \\
\hline History of CVD (\%) & 35 & 32 & 40 & None \\
\hline BMI $\left(\mathrm{kg} / \mathrm{m}^{2}\right)$ & 32 & 28 & 31 & SI:29; M:32 \\
\hline Mean baseline $\mathrm{HbA}_{\mathrm{Ic}}(\%)$ & 8.1 & 7.5 & 9.4 & SI:7.9; M:8,4 \\
\hline On insulin at baseline (\%) & 35 & 1.5 & 52 & 64 \\
\hline \multicolumn{5}{|l|}{ Protocol characteristics } \\
\hline $\mathrm{HbA}_{\mathrm{Ic}}$ goals $(\%)(\mathrm{Ivs} S)^{\mathrm{a}}$ & $<6.0$ vs $7.0-7.9$ & $\begin{array}{l}\leq 6.5 \text { vs 'based on } \\
\text { local guidelines' }\end{array}$ & $\begin{array}{l}<6.0 \text { (action if }>6.5) \\
\text { vs planned separation } \\
\text { of } 1.5\end{array}$ & $\begin{array}{l}\text { Aiming for fasting plasma } \\
\text { glucose }<6 \mathrm{mmol} / \mathrm{L}\end{array}$ \\
\hline $\begin{array}{l}\text { Protocol for glycemic } \\
\text { control (I vs S) }\end{array}$ & $\begin{array}{l}\text { Multiple drugs in } \\
\text { both arms }\end{array}$ & $\begin{array}{l}\text { Multiple drugs } \\
\text { added to gliclizide vs } \\
\text { multiple drugs with } \\
\text { no gliclizide }\end{array}$ & $\begin{array}{l}\text { Multiple drugs in } \\
\text { both arms }\end{array}$ & $\begin{array}{l}\text { Intensive therapy (either } \\
\text { sulfonylurea or insulin/SI } \\
\text { or, min overweight } \\
\text { patients, metformin/M) vs } \\
\text { corresponding conventional } \\
\text { therapy group/C (dietary } \\
\text { restriction) }\end{array}$ \\
\hline Management of risk factors & $\begin{array}{l}\text { Embedded BP and } \\
\text { lipid trials }\end{array}$ & Embedded BP trial & $\begin{array}{l}\text { Protocol for } \\
\text { intensive treatment } \\
\text { in both arms }\end{array}$ & None \\
\hline \multicolumn{5}{|l|}{ On study characteristics } \\
\hline Median duration of follow-up (years) & 3.5 (terminated early) & 5 & 5.6 & 8.8 \\
\hline Achieved median $\mathrm{HbA}_{\mathrm{Ic}}(\%)(\mathrm{I} \text { vs S})^{\mathrm{a}}$ & 6.4 vs 7.5 & 6.4 vs 7.0 & 6.9 vs 8.5 & SI:7.9 vs $7.9 ; \mathrm{M}: 8.1$ vs 8.1 \\
\hline On insulin at study end (\%) (I vs S $)^{a}$ & 77 vs $55^{a}$ & 40 vs 24 & 89 vs 74 & Data not shown \\
\hline On TZD at study end (\%) (I vs S) & 91 vs $58^{a}$ & I7 vs II & 53 vs 42 & None \\
\hline On statin at study end (\%) (I vs S $)^{a}$ & 88 vs $88^{a}$ & 46 vs 48 & 85 vs 83 & Data not shown \\
\hline On aspirin at study end (\%) (I vs S $)^{\mathrm{a}}$ & 76 vs $76^{a}$ & 57 vs 55 & 88 vs 86 & Data not shown \\
\hline Smokers at study end (\%) & 10 & 8 & 8 & Data not shown \\
\hline \multicolumn{5}{|l|}{$\begin{array}{l}\text { Mean blood pressure at study } \\
\text { end }(\mathrm{mmHg})\end{array}$} \\
\hline Intensive glycemic control arm & $126 / 67$ & $136 / 74$ & $127 / 68$ & Data not shown \\
\hline Standard glycemic control arm & $127 / 68$ & $138 / 74$ & $125 / 69$ & Data not shown \\
\hline \multicolumn{5}{|l|}{ Weight changes (kg) } \\
\hline Intensive glycemic control arm & +3.5 & -0.1 & +7.8 & SI: +1.0 \\
\hline Standard glycemic control arm & +0.4 & -1.0 & +3.4 & M:-1.0 \\
\hline \multicolumn{5}{|l|}{$\begin{array}{l}\text { Severe hypoglycemia } \\
\text { participants with I or more } \\
\text { episodes during study (\%) }\end{array}$} \\
\hline Intensive glycemic control arm & 16.2 & 2.7 & 21.2 & Data not shown \\
\hline Standard glycemic control arm & 5.1 & 1.5 & 9.9 & Data not shown \\
\hline
\end{tabular}


Table I (Continued)

\begin{tabular}{|c|c|c|c|c|}
\hline & ACCORD & ADVANCE & VADT & UKPDS post-trial \\
\hline \multicolumn{5}{|l|}{ Outcomes } \\
\hline Definition of primary outcome & $\begin{array}{l}\text { Nonfatal MI, nonfatal } \\
\text { stroke, CVD death }\end{array}$ & $\begin{array}{l}\text { Microvascular plus } \\
\text { macrovascular (nonfatal } \\
\text { MI, nonfatal stroke, CVD } \\
\text { death) outcomes }\end{array}$ & $\begin{array}{l}\text { Nonfatal MI, nonfatal } \\
\text { stroke, CVD death, } \\
\text { hospitalization for heart } \\
\text { failure, revascularization }\end{array}$ & $\begin{array}{l}\text { Any diabetes-related } \\
\text { endpoint, diabetes-related } \\
\text { death, death from } \\
\text { any cause, MI, stroke, } \\
\text { peripheral vascular disease, } \\
\text { microvascular disease }\end{array}$ \\
\hline HR for primary outcome $(95 \% \mathrm{Cl})$ & $0.90(0.78-1.04)$ & $\begin{array}{l}0.9(0.82-0.98) \\
\text { macrovascular } 0.94 \\
(0.84-1.06)\end{array}$ & $0.88(0.74-1.05)$ & $\begin{array}{l}\text { SI:0.9I (0.83-0.99); } \\
\text { M:0.79 (0.66-0.95) }\end{array}$ \\
\hline HR for mortality findings $(95 \% \mathrm{Cl})$ & $1.22(1.01-1.46)$ & $0.93(0.83-1.06)$ & $\mathrm{I} .07(0.8 \mathrm{I}-\mathrm{I} .42)$ & $\begin{array}{l}\text { SI:0.87 (0.79-0.96); } \\
\text { M:0.73 (0.59-0.89) }\end{array}$ \\
\hline
\end{tabular}

"Medication rates for ACCORD are for any use during the study.

Abbreviations: AC, active control; BMI, body mass index; BP, blood pressure; C, conventional therapy; CVD, cardiovascular disease; I, intensive glycemic control; M, metformin group; MI, myocardial infarction; PC, placebo control; RCT, randomized controlled trial; S, standard glycemic control; SI, sulfonylurea-insulin group; TZD, thiazolidinedione.

glycemia between the treatment groups or the different profile of medications utilized to achieve the glycemic levels was responsible for the excess mortality. 5,9

\section{ADVANCE}

The ADVANCE trial was designed to evaluate the effects of intensive glycemic and BP control on CVD in patient with long-standing T2DM at high risk for vascular disease. ${ }^{5,10}$ The primary outcome endpoint was a combination of microvascular events (nephropathy and retinopathy) and major adverse cardiovascular events (MI, stroke, and cardiovascular death). ${ }^{5,10}$ The results of the BP-lowering arm are discussed further below.

In the intensive glucose lowering arm, 11,140 T2DM patients at high risk of CVD (mean age 66 years, mean duration of diabetes eight years) were randomly assigned to modified-release gliclazide (30 to $120 \mathrm{mg}$ ), plus other drugs as required, to achieve an $\mathrm{HbA}_{1 \mathrm{c}}$ of $<6.5 \%$ or to standard therapy (in which any medication but gliclizide could be used, with the glycemic target set according to "local guidelines"). ${ }^{5,10}$ Compared to ACCORD participants, patients with T2DM in ADVANCE were slightly older but of similar high CVD risk, the average duration of diabetes was about 2 years shorter, the baseline $\mathrm{HbA}_{1 \mathrm{c}}$ (median 7.2\%) was lower whereas almost no insulin was used at enrolment. ${ }^{5,10}$ The primary endpoint was significantly reduced in the intensive glucose lowering arm (HR 0.90 [95\% CI 0.82 to 0.98 ], $P=0.01$ ), although this was only due to a significant reduction of microvascular diabetic nephropathy classifed as development of macroalbuminuria (0.86 [0.77 to 0.97], $P=0.01)$, with no significant decline in the macrovascular outcome (0.94 [0.84 to 1.06 ], $P=0.32) .^{5,10}$
After a median of 5 years of follow-up, the intensive and standard groups achieved mean $\mathrm{HbA}_{1 \mathrm{c}}$ values of 6.5 and $7.3 \%$, respectively, with the $\mathrm{HbA}_{1 \mathrm{c}}$ in the intensive group on average $0.67 \%$ lower. ${ }^{5,10}$ Severe hypoglycemia occurred in more patients in the intensively treated arm (2.7 vs $1.5 \%) .5,10$ Mean body weight was $0.7 \mathrm{~kg}$ greater in the intensive compared with standard therapy group. ${ }^{5,10}$ Unlike the findings described above in the ACCORD trial, ADVANCE did not show an increased risk of death among patients receiving intensive therapy compared with standard therapy (HR for death from cardiovascular causes with intensive control $0.88,95 \%$ CI 0.74 to 1.04 ; HR for death from any cause $0.93,95 \%$ CI 0.83 to 1.06). ${ }^{5,10}$ As in ACCORD, there was no benefit of intensive therapy on the primary composite endpoint of cardiovascular death, nonfatal MI, or nonfatal stroke (HR $0.94,95 \%$ CI 0.84 to 1.06 ) although the use of other cardioprotective drugs such as aspirin, statins or angiotensin-converting enzyme inhibitor (ACEI) was even lower in ADVANCE than in ACCORD or VADT. ${ }^{5,10}$

\section{VADT}

In the prospective VADT, investigators randomized 1791 military veterans with T2DM (mean age 60 years, mean duration 11.5 years, suboptimal response insulin or maximal-dose oral agents or standard glucose control) to a strategy of intensive glycemic control (goal $\mathrm{HbA}_{1 \mathrm{c}}<6.0 \%$ ) or standard glycemic control (planned $\mathrm{HbA}_{1 \mathrm{c}}$ separation $>1.5 \%$ ). ${ }^{11}$ At the time of randomization, median $\mathrm{HbA}_{1 \mathrm{c}}$ levels were 9.4\% while nearly $75 \%$ of patients had hypertension and $40 \%$ had a previous cardiovascular event. ${ }^{11}$

A variety of agents (metformin, glimepiride, rosiglitazone, insulin) were used to achieve glycemic goals. ${ }^{11}$ In both 
study groups, obese patients were started on 2 drugs, metformin and rosiglitazone, whereas nonobese patients were started with glimepiride plus rosiglitazone. ${ }^{11}$ Patients in the intensive arm started on maximal doses. ${ }^{11}$ Insulin was added to most participants to achieve $\mathrm{HbA}_{1 \mathrm{c}}$ levels less than $6.0 \%$ in the intensive-treatment arm and less than $9.0 \%$ in the standard-therapy arm. ${ }^{11}$ Furthermore, aggressive BP, high levels of aspirin and statin usage, and a high degree of smoking cessation was acheived in both study groups. ${ }^{11}$

The primary outcome of VADT was a composite of CVD events (MI, stroke, cardiovascular death, revascularization, hospitalization for heart failure, and amputation for ischemia). ${ }^{5}$ After a median follow-up of 6.5 years, there was no significant difference in the first occurrence of any cardiovascular event (composite of stroke, death from cardiovascular causes, chronic heart failure, surgery for vascular disease, inoperable coronary disease, amputation for ischemic gangrene) between the intensive (achieved $\left.\mathrm{HbA}_{1 \mathrm{c}} 6.9 \%\right)$ and standard $\left(\mathrm{HbA}_{1 \mathrm{c}} 8.4 \%\right)$ groups (HR in the intensive group $0.88,95 \%$ CI 0.74 to 1.05$).{ }^{11}$ In addition, there was no difference between groups in time to death from cardiovascular causes (HR 1.32, 95\% CI 0.81 to 2.14) or death from any cause (HR 1.07, 95\% CI 0.81 to 1.42 ). ${ }^{11}$ Hypoglycemia occurred more frequently in the intensive group ( $8.5 \%$ vs $3.1 \%$ ), including episodes with impaired or complete loss of consciousness (12 vs 4 per 100 patient-years). ${ }^{11}$ Post hoc subgroup analyses further indicated that participants with duration of diabetes $<12$ years appeared to have a CVD benefit of intensive glycemic control whereas participants with loger duration of diabetes might experience even adverse effect of intensive glycemic control. ${ }^{11}$ Other exploratory analyses suggested severe hypoglycemia within the last 3 months as a predictor of CVD mortality, with an association of severe hypoglycemia with all-cause mortality apparent only for participants in the standard arm. ${ }^{5}$

\section{UKPDS post-trial study}

The original UKPDS was designed to investigate the role of glycemic control on the complications of T2DM in newly diagnosed patients. ${ }^{6}$ There was no difference in macrovascular disease in the intensive and conventional therapy groups (mean $\mathrm{HbA}_{1 \mathrm{c}}$ values of $7 \%$ and $7.9 \%$, respectively) in the primary analysis. ${ }^{6}$ A subset of 753 overweight patients (mean BMI $31 \mathrm{~kg} / \mathrm{m}^{2}$ ) was included in a separate treatment arm in which intensive blood glucose control with metformin was compared with conventional therapy. ${ }^{6}$ A secondary analysis compared the 342 patients allocated to metformin with 951 patients receiving intensive therapy with a sulfonylurea or insulin. ${ }^{6}$ Compared to conventional therapy, treatment with metformin resulted in significant risk reductions of $32 \%$ for any diabetesrelated endpoint (endpoints included both macrovascular and microvascular complications), $42 \%$ for diabetes-related death, and 36\% for all-cause mortality. ${ }^{6}$ Metformin also had a greater effect upon any diabetes-related endpoint and allcause mortality than intensive therapy with a sulfonylurea or insulin $(P=0.003){ }^{6}$

Novel data from the UKPDS come from post-trial monitoring to follow up those patients either on conventional therapy (dietary restriction) or intensive therapy (either sulfonylurea or insulin or, in overweight patients, metformin) for a further 10 years, giving an overall median follow-up for the glucose control comparison of about 17 years. ${ }^{12}$ For the first 5 years of the post-trial monitoring, 3,227 patients were asked to attend annual UKPDS clinics but no attempts were made to maintain their previously assigned therapies. ${ }^{12}$ In years 6 to 10 of this further follow-up, all patients were assessed only through questionnaires. ${ }^{12}$ The long-term clinical outcomes were assessed, according to the initial randomization, on an intention-to-treat basis. ${ }^{12}$

Notably, between-group differences in $\mathrm{HbA}_{1 \mathrm{c}}$ levels were lost within one year of stopping the randomly assigned therapies. ${ }^{12}$ However, a surprising "legacy effect" emerged for both the sulfonylurea/insulin and the metformin groups, with significant reductions observed in MI and and in all-cause mortality in the intensive glycemic control group. ${ }^{12}$ In the sulfonylurea/insulin group, relative reductions in risk persisted at 10 years for any diabetes-related outcomes $(9 \%$, $P=0.04)$, and microvascular disease $(24 \%, P=0.001)$ and risk reductions emerged over time for diabetes-related deaths $(17 \%, P=0.01)$, MI $(15 \%, P=0.01)$ and death from any cause $(13 \%, P=0.007)(12)$. In the metformin group, significant reductions persisted for any diabetes-related endpoint $(21 \%, P=0.01), \operatorname{MI}(33 \%, P=0.005)$ and all-cause mortality $(27 \%, P=0.002) .{ }^{12}$

In summary, these results indicate that a sustained period of glycemic control over ten years in newly diagnosed patients with T2DM has a long-term benefit in reducing cardiovascular morbidity and mortality. The results are supported by similar findings from the postinterventional follow-up of subjects enrolled in the Steno- 2 trial. ${ }^{4}$

Notably, in the above-mentioned glucose-lowering trials, other CVD risk factors were treated to a moderate or high degree, probably causing lower rates of CVD in the standard arm than originally predicted. ${ }^{5}$ Furthermore, most patients received multiple glucose-lowering drugs with distinct 
pharmacological properties, either with or without insulin, to acheive blood glucose control in all studies. However, whereas in the ACCORD study glucose-lowering treatments to reach glycemic targets were not restricted to a single drug class, the ADVANCE study required sulfonylurea gliclazide (modified release) for all patients in the intensive-control group at least at initiation. ${ }^{5}$ While further comparing the ACCORD and ADVANCE studies, thiazolidinedione treatment was more frequently prescribed in the intensive-therapy group of the ACCORD trial which may interfere with the increase in mortality in ACCORD. ${ }^{5}$ As it will be further outlined below, both trials used a factorial design to test additional and different treatment interventions in their study participants. Participants of the ACCORD study were randomly assigned to undergo intensive therapy or standard therapy for BP lowering or to receive fenofibrate or placebo, whereas patients of the ADVANCE study were randomly assigned to receive a combination of perindopril and indapamide or to receive placebo. ${ }^{14}$ Surprisingly, neither study appears to have emphasized lifestyle or dietary modification - the solid rock of any treatment strategy in T2DM.

Most previous reviewers agree that the increase in mortality in ACCORD is probably due to the overall treatment strategies for intensifying glycemic control in the study population and not the achieved $\mathrm{HbA}_{1 \mathrm{c}}$ per se. ${ }^{5}$ In this context, it is important to emphasize that also diabetic patients in intensive arm of the ADVANCE study achieved a median $\mathrm{HbA}_{1 \mathrm{c}}$ similar to that from patients of the ACCORD study while no increased mortality hazard was observed in the former study. ${ }^{5}$ Consequently, the increased mortality findings from the ACCORD study do not mean that patients with $\mathrm{T} 2 \mathrm{DM}$ with low $\mathrm{HbA}_{1 \mathrm{c}}$ levels due to lifestyle modifications or pharmacotherapy are at risk or even need to augment their $\mathrm{HbA}_{1 \mathrm{c}} \cdot{ }^{5}$ Notably, the unfavorable cardiac effects of hypoglycemia, which are probably induced by a complex interplay of its overactivation of the sympathetic nervous system and cardiac dysrhythmia, have been mainly shown in T2DM with CVD or at high risk of sudden cardiac death and ventricular arrhythmias. ${ }^{15}$ It is biologically plausible that once an event occurs, if the current glycemic control is too tight, this may predict an unfavorable outcome in view of the known detrimental cardiac effects of hypoglycemic episodes and events. ${ }^{15}$ Furthermore, hypoglycemia unawareness probably due to diabetic autonomic neuropathy may aggravate this situation. ${ }^{15}$ Unfortunately, lethal hypoglycemic events are often erroneously considered as fatal coronary artery disease, mainly due to insufficient diagnosis criteria postmortem. ${ }^{5}$
If hypoglycemia was indeed a contributing cause of death in the ACCORD trial, future studies of cardiovascular risk reduction should focus on achieving near-normal glycemic levels with the use of strategies and therapies associated with a lower risk of hypoglycemia.

Other plausible mechanisms for the increase in mortality in the ACCORD study population with advanced T2DM and multiple comorbidities include weight gain, distinct drug effects or interactions, or the use of multiple oral glucoselowering drugs along with multiple doses of insulin to rapidly acheive very low $\mathrm{HbA}_{1 \mathrm{c}}$ targets. ${ }^{5}$ The recent findings from the UKPDS trial adds to this picture that glycemic control early in the course of T2DM may have CVD benefit which has been defined as "metabolic memory" or "legacy effect". ${ }^{5,16}$ As is the case with microvascular complications, it may be that glycemic control plays a greater role before macrovascular disease is well developed and a minimal or no role when this detrimental complication is already advanced. ${ }^{5}$

In summary, although the evidence for CVD prevention by statin therapy, BP treatment, aspirin therapy in high-risk patients, and other interventions is robust, ${ }^{5}$ data on the effects of antihyperglycemic therapies are less evident. In T2DM, when other CVD risk factors are highly prevalent, the additive benefits of intensive glycemic control might be difficult to demonstrate except in even larger or longer trials. ${ }^{5}$ It is likely that a real benefit of glucose lowering on CVD in T2DM, even if it could be proven, is modest compared with and incremental to treatment of other established CVD risk factors. ${ }^{5}$

\section{The beat is on: blood pressure control in T2DM patients}

BP is an important determinant of the risk of macro- and microvascular vascular complications in patients with T2DM. Current European guidelines for the management of hypertension recommend lowering BP in patients with T2DM to reduce the risk of cardiovascular events. ${ }^{17}$ Guidelines also recognize the relevance of prediabetes, a situation characterized by the presence of the metabolic syndrome and consider the need for integral protection of the cardiovascular and renal systems that includes a goal BP lower than 130/80 mmHg, accompanied by strict metabolic and lipid control often accompanied by antiplatelet therapy. ${ }^{18}$ Furthermore, a reduction in the frequency of diabetic nephropathy by ACEI inhibitor treatment in normotensive lean microalbuminuric patients T2DM has been shown either. ${ }^{19}$ More recently, also beneficial effects with ARBs in hypertensive patients with T2DM and nephropathy have been demonstrated. ${ }^{20}$ In order 
to attain the adequate BP and cardiorenal protection beyond the BP decrease, suppression of the renin-angiotensin system (RAS) should therefore be attempted, usually in association with other antihypertensive drugs. ${ }^{18}$ Recently, the ONgoing Telmisartan Alone and in combination with Ramipril Global Endpoint Trial (ONTARGET) compared the ACEI ramipril (10 $\mathrm{mg} /$ day), the angiotensin receptor blocker (ARB) telmisartan $80 \mathrm{mg} /$ day, and the combination of the 2 drugs in 25,620 patients with vascular disease or high-risk diabetes. ${ }^{21}$ After a median follow up of 56 months, no significant differences were observed between the 3 groups either in the primary composite outcome (death from cardiovascular causes, MI, stroke, or hospitalization for heart failure), or in each of its components, total mortality and other secondary outcomes. ${ }^{21}$ Telmisartan was equivalent to ramipril (noninferiority criterion), but was better tolerated (less cough and angioedema). ${ }^{21}$ The combination of the 2 drugs in this population (without congestive heart failure and proteinuric nephropathy), however, did not bring increased benefit (no superiority), but was associated with more adverse events (hypotension, syncope and renal dysfunction). ${ }^{21}$ In this population, the choice of the molecule in monotherapy remains optional and the use of a dual blockade is not justified in order to have a better cardiovascular protection. ${ }^{21}$ Diuretics are often used as an adjunctive to reduce BP and form a very important basis for antihypertensive treatment, also often in combination with agents that inhibit the RAS. ${ }^{22,23}$ Several studies demonstrated that treatment with the diuretic indapamide reduces the level of microalbuminuria in patients with T2DM. ${ }^{22,23}$ It is therefore understandable that many guidelines suggest that diuretics form part of the treatment of hypertension in patients with T2DM. ${ }^{17}$ However, adequate BP targets as indicated above cannot usually be reached using monotherapies especially in patients who present with a high cardiovascular risk such as T2DM. ${ }^{18}$

Thus, is the lower the better for BP control in T2DM patients or should we doubt such a conclusion in the light of increased mortality in the ACCORD trial? It is noteworthy to mention that clinical trials have reported a difference of 5 to $6 \mathrm{mmHg}$ between the diastolic BP of treatment and control groups; the risk reduction of $14 \%$ (95\% CI $4 \%$ to $22 \%$ ) for coronary events contrasts with the $20 \%$ to $25 \%$ reduction noted in epidemiologic reports. ${ }^{19}$ These discrepancies may be related to the existence of a J-shaped relationship between BP and risk, in which treated patients with low BP are at increased risk for coronary events. ${ }^{19}$ This effect is probably due to compromised coronary blood flow, especially in hypertensive patients with a history of MI, or to other factors independent from treatment with BP-reducing drugs such as deteriorating heath or pulse pressure. ${ }^{19}$ However, major epidemiological studies such as Framingham and the Multiple Risk Factor Intervention Trial (MRFIT) Diabetic Cohort showed that the J-shaped relationship between BP and mortality may not be so relevant. ${ }^{19,24,25}$ The latter study demonstrated that systolic BP and complications are clearly associated in diabetes patients without any threshold value while a 2- to 4-fold increase in cardiovascular mortality was observed. ${ }^{19,25}$ The UKPDS trial demonstrated that tight $\mathrm{BP}$ control $(<150 / 85 \mathrm{mmHg})$ resulted in a risk reduction compared with the less tight control arm $(<180 / 105 \mathrm{mmHg}){ }^{26}$ Reductions in risk in the group assigned to tight control compared with that assigned to less tight control were $24 \%$ in diabetes related endpoints (95\% CI $8 \%$ to $38 \%$ ) $(P=0.0046), 32 \%$ in deaths related to diabetes $(6 \%$ to $51 \%)$ $(P=0.019), 44 \%$ in strokes $(11 \%$ to $65 \%)(P=0.013)$, and $37 \%$ in microvascular end points $(11 \%$ to $56 \%)(P=0.0092) .{ }^{26}$ There was a nonsignificant reduction in all-cause mortality. ${ }^{26}$ In the tight control group, $29 \%$ needed 3 or more antihypertensive drugs. ${ }^{26}$ After 9 years of follow up, $29 \%$ of patients in the group assigned to tight control required 3 or more treatments to lower BP to achieve target BP. ${ }^{26}$ The HOT (Hypertension Optimal Treatment) study showed that the frequency of major CVD events (11.9/1000 patients/year) is significantly reduced in the group with a diastolic BP target $<80 \mathrm{mmHg}$ (achieved $144 / 81 \mathrm{mmHg}$ ) was $11.9 / 1000$ patients/year compared to the event rate $(24.4 / 1000$ patients/year) in the group with target $<90 \mathrm{mmHg}$ (achieved 148/85 $\mathrm{mmHg}$ ). ${ }^{27}$ Thus, there is now increasing evidence that BP should be as low as possible in patients with T2DM.

A few months after the publication of the last European guidelines, ${ }^{17}$ the hypertension branch of the ADVANCE study was published. ${ }^{14}$ As it is agreed that multifactorial treatment regimens are required to reduce the cardiovascular burden in patients with T2DM, the ADVANCE study not only investigated the potential benefits of tighter glucose control (see above) but also the potential benefits of BP lowering, using the routine administration of an ACEI/diuretic combination (fixed low-dose combination of perindopril and indapamide vs placebo) irrespective of initial BP levels or the use of other BP lowering drugs. ${ }^{14}$ The two primary outcomes, taken separately and jointly, are a composite macrovascular endpoint of nonfatal stroke, nonfatal MI and cardiovascular death, and a composite microvascular endpoint of new or worsening nephropathy or microvascular eye disease. After a 6-week active run-in period, 11,140 patients with T2DM were randomized to treatment with a fixed combination of perindopril and 
indapamide or matching placebo (mean of 4.3 years of follow-up), in addition to current therapy. ${ }^{14}$ Compared with patients assigned placebo, those assigned active therapy had a mean reduction in systolic BP of $5.6 \mathrm{mmHg}$ and diastolic BP of $2.2 \mathrm{mmHg} .{ }^{14}$ The relative risk of a major macrovascular or microvascular event was reduced by $9 \%(861$ [15.5\%] active vs 938 [16.8\%] placebo; HR $0.91,95 \%$ CI 0.83 to $1.00, P=0.04) .{ }^{14}$ The separate reductions in macrovascular and microvascular events were similar but were not independently significant (macrovascular $0.92 ; 0.81$ to 1.04 , $P=0.16$; microvascular $0.91 ; 0.80$ to $1.04, P=0.16) .{ }^{14}$ The relative risk of death from CVD was reduced by $18 \%$ (211 [3.8\%] active vs 257 [4.6\%] placebo; 0.82, 0.68-0.98, $P=0.03)$ and death from any cause was reduced by $14 \%$ (408 [7.3\%] active vs 471 [8.5\%] placebo; $0.86,0.75$ to $0.98, P=0.03) .{ }^{14}$ There was no evidence that the effects of the study treatment differed by initial BP level or concomitant use of other treatments at baseline. ${ }^{14}$ In conclusion, this study has shown that the addition of a fixed combination of perindopril and indapamide on top of the other medications in patients with diabetes with $\mathrm{BP}$ ranging from normal to elevated greatly helps diminish BP when required, while protecting the cardiovascular and renal systems from the deleterious effects of diabetes associated with high BP. ${ }^{14}$ This protection includes as the most relevant finding a significant decrease in all-cause death. ${ }^{14}$ Although the confidence limits were wide, the results suggest that over 5 years, 1 death due to any cause would be averted among every 79 patients assigned active therapy. ${ }^{14}$

Lately, the post-trial monitoring of patients in the UKPDS also examined whether risk reductions for microvascular and macrovascular disease, achieved with the use of improved BP control during the trial, would be sustained. ${ }^{28}$ Intriguingly, the benefits of previously improved BP control were not sustained when between-group differences in BP were lost. ${ }^{28}$ Thus, in contrast to the "metabolic memory" in the diabetic state, no "legacy effect" has been observed for hypertension. ${ }^{28}$

In conclusion, BP targets should aimed to be as low as possible in patients with T2DM. Furthermore, early improvement in BP control in patients with both T2DM and hypertension was associated with a reduced risk of complications, but it appears that good BP control must be continued if the benefits are to be maintained.

\section{Implications for clinical care of T2DM patients}

Intensive glycemic control is well established to avoid on microvascular and neuropathic complications in both
T1DM and T2DM. ${ }^{5}$ The ADVANCE trial has added to that evidence base by demonstrating a significant reduction in the risk of new or worsening albuminuria when median $\mathrm{HbA}_{1 \mathrm{c}}$ was lowered to $6.3 \%$ compared with standard glycemic control achieving an $\mathrm{HbA}_{1 \mathrm{c}}$ of 7.0\%. The lack of significant reduction in CVD events with intensive glycemic control in ACCORD, ADVANCE, and VADT, however, should not lead clinicians to abandon the general target of an $\mathrm{HbA}_{1 \mathrm{c}}<7.0 \%$ and thereby disregard the benefit of adequate blood glucose control on devastating microvascular complications. ${ }^{5}$

The ADA's Standards of Medical Care in Diabetes ${ }^{29}$ and the American Heart Association (AHA) and ADA's scientific statement on prevention further advocate controlling nonglycemic risk factors (through BP control, lipid lowering with statin therapy, aspirin therapy and lifestyle modifications) as the primary strategies for reducing the burden of CVD in people with T2DM. ${ }^{30}$ The lower-than-predicted CVD rates in ACCORD, ADVANCE and VADT, as well as the recent long-term followup of the Steno-2 multiple risk factor intervention, ${ }^{4}$ provide strong evidence that optimal treatment for T2DM involves targeting of all vascular risk factors and not just hyperglycemia alone. ${ }^{5}$ The evidence for a cardiovascular benefit of intensive glycemic control remains strongest for those with T1DM.,8 However, subset analyses of ACCORD, ADVANCE, and VADT suggest the hypothesis that patients with shorter duration of T2DM and without established atherosclerosis may also achieve cardiovascular benefit from intensive glycemic control..$^{5}$ In contrast, potential risks of intensive glycemic control may outweigh its benefits in other T2DM patients, eg, with long disease duration, severe hypoglycemia, present atherosclerosis and advanced age/frailty. ${ }^{5}$ It is suggested that prevention of severe hypoglycemia in T2DM patients with advanced disease should be placed in the spotlight and aggressive normalization to near-normal $\mathrm{HbA}_{1 \mathrm{c}}$ levels should be avoided in such patients..$^{5}$ In conclusion, the evidence obtained from ACCORD, ADVANCE, and VADT does not suggest the need for major changes in glycemic control targets but, rather, additional clarification of the language that has consistently stressed individualization as stated in more detail lately in a position statement of the ADA and a scientific statement of the American College of Cardiology (ACC) Foundation and the AHA: ${ }^{5}$

- Microvascular disease: Lowering $\mathrm{HbA}_{1 \mathrm{c}}$ to below or around $7 \%$ has been shown to reduce microvascular and neuropathic complications of T1DM and T2DM. Therefore, the $\mathrm{HbA}_{1 \mathrm{c}}$ goal for nonpregnant adults in general is $<7 \%$. ADA, A-level recommendation; ACC/AHA, class I recommendation (level of evidence $\mathrm{A}$ ). 
- Macrovascular disease: In T1DM and T2DM, randomized controlled trials of intensive vs standard glycemic control have not shown a significant reduction in CVD outcomes during the randomized portion of the trials. However, long-term follow-up of the DCCT and UKPDS cohorts suggests that treatment to $\mathrm{HbA}_{1 \mathrm{c}}$ targets below or around $7 \%$ in the years soon after the diagnosis of diabetes is associated with long-term reduction in risk of macrovascular disease. Until more evidence becomes available, the general goal of $<7 \%$ appears reasonable. ADA, B-level recommendation; ACC/AHA, class IIb recommendation (level of evidence $\mathrm{A}$ ).

For some patients, individualized glycemic targets other than the above general goal may be appropriate as stated by Skyler et al: ${ }^{5}$

- Subgroup analyses of clinical trials such as the DCCT and UKPDS and the microvascular evidence from the ADVANCE trial suggest a small but incremental benefit in microvascular outcomes with $\mathrm{HbA}_{1 \mathrm{c}}$ values closer to normal. Therefore, for selected individual patients, providers might reasonably suggest even lower $\mathrm{HbA}_{1 \mathrm{c}}$ goals than the general goal of $<7 \%$ if this can be achieved without significant hypoglycemia or other adverse effects of treatment. Such patients might include those with short duration of diabetes, long life expectancy, and no significant CVD. ADA, B-level recommendation; ACC/AHA, class IIa recommendation (level of evidence $\mathrm{C}$ ).

- Conversely, less stringent $\mathrm{HbA}_{1 \mathrm{c}}$ goals than the general goal of $<7 \%$ may be appropriate for patients with a history of severe hypoglycemia, limited life expectancy, advanced microvascular or macrovascular complications, or extensive comorbid conditions or those with long-standing diabetes in whom the general goal is difficult to attain despite diabetes self-management education, appropriate glucose monitoring, and effective doses of multiple glucose-lowering agents including insulin. ADA, C-level recommendation; ACC/AHA, class IIa recommendation (level of evidence C).

For primary and secondary CVD risk reduction in patients with diabetes, providers should continue to follow the evidence-based recommendations for BP treatment, including lipid-lowering with statins, aspirin prophylaxis, smoking cessation and healthy lifestyle behaviors delineated in the ADA Standards of Medical Care in Diabetes ${ }^{29}$ and the AHA/ADA guidelines for primary CVD prevention. ${ }^{30}$

\section{The patient's viewpoint}

As newer oral diabetes agents continue to emerge on the market, comparative evidence is urgently required to guide appropriate therapy. ${ }^{31} \mathrm{~A}$ meta-analysis of the recent literature stated that compared with newer, more expensive agents (incretin, mimetics, thiazolidinediones, alpha-glucosidase inhibitors, meglitinides), the longer-established agents such as second-generation sulfonylureas and metformin have similar or superior effects on glycemic control, lipids, and other intermediate endpoints. ${ }^{31}$ Developments and refinements within these classes have included the introduction of modified-release preparations, and the emergence of fixed-dose preparations with metformin and with novel drugs. ${ }^{32}$ The former include the thiazolidinediones, agents with a putative genomic mechanism of action that have been under continuous observation due to severe hepatotoxicity with the compound troglitazone as well as the issue of edema and the risk of precipitating heart failure in vulnerable patients. ${ }^{32}$ Rapid-acting nonsulfonylurea secretagogues appear to be effective and perhaps safer than sulfonylureas in some groups of patients with certain comorbidities (eg, those with renal impairment). ${ }^{32}$ Alphaglucosidase inhibitors have an excellent safety record and acarbose has been shown to retard the progression from impaired glucose tolerance to T2DM. ${ }^{32}$ However, their general use is limited by tolerability issues.

It is noteworthy that the conventional wisdom falsely assumes that the clinician's key focus ought to be on reducing risk factors below specific levels. ${ }^{33}$ This approach, however, still neglects the importance of which specific strategies are used to modify these factors as recently discussed eagerly in an editorial article from Krumholz et al. ${ }^{33}$ It is stated that the risk/benefit ratio of interventions designed to modify risk factors can vary depending on the type and number of medications and other approaches that are concurrently incorporated. ${ }^{33}$ The authors assume that some medications may even have beneficial or harmful effects beyond their effect on a risk factor. ${ }^{33}$ Nowadays, also the US Food and Drug Administration and the European Medicines Agency focus more and more on patient outcomes rather than intermediate or surrogate markers as some therapeutic strategies. ${ }^{33}$ In the future, therefore, doctors will have to appreciate that improvement of risk factor levels or other intermediate outcomes may not necessarily predict its effect on individual patient outcomes. ${ }^{33}$ In the general interest of promoting good care in patients with T2DM, national guidelines and performance measures have been constructed that encourage treatment geared toward achieving ambitious goals for levels of $\mathrm{HbA}_{1 \mathrm{c}}$, lipids, and $\mathrm{BP}{ }^{33}$ It is important, however, to realize that these treatment goals generally do not specify the strategy behind to acheive a treatment target. ${ }^{33}$ Future clinical studies therefore 
will have to consider treatment strategies and performance measures appreciating the assessment of net clinical benefit based on events averted or lives improved. ${ }^{33}$ The promulgation of those strategies that are shown to be effective will serve as an incentive for drug and device developers to provide evidence about patient outcomes, not just about how a drug or device affects intermediate outcomes. ${ }^{33}$ Moving practice toward evidence-based strategies must ensure that in implementing quality measures we are always acting in the patient's best interests ('individualized medicine'). ${ }^{33}$ The recently published randomized control trials in T2DM, namely ACCORD, ADVANCE, VADT and UKPDS posttrial, demonstrate the complexity of interpretation of clinical trails and that we need to understand a strategy's effects on people, not just on surrogate endpoints.

\section{Conclusions}

The most compelling message from all recent cardiovascular studies in patients with T2DM is that near-normal glycemic control for a median of 3.5 to 5 years does not reduce cardiovascular events within that time frame. However, the ADVANCE trial reconfirmed the predicted reductions in new-onset microalbuminuria and nephropathy observed in the UKPDS. ${ }^{34}$ A troubling finding from the ACCORD trial is that near-normal glucose control (achieved with the use of combination therapy incorporating heavy use of thiazolidinediones, sulfonylureas, metformin and insulin) is associated with significantly increased risks of death from any cause and death from cardiovascular causes, the very outcomes the trial was designed to prevent.

The results of the ACCORD and ADVANCE studies should be interpreted in the context of comprehensive care of patients with T2DM. There is clear evidence that aspirin, statins and the targeted lowering of BP are each associated with substantial reductions in cardiovascular risk in patients with T2DM; there may be even greater benefit when these reductions are achieved together. ${ }^{3,4}$ The associated therapies are evidence-based, widely endorsed, and worthwhile but can be difficult, time-consuming and resource intensive to implement, even when care is provided in a dedicated diabetes center. Before new targets are defined, it is worth reflecting that the currently established targets for hyperglycemia, hypertension and hyperlipidemia are achieved only in few patients $(<10 \%) .{ }^{29,30}$ Clinicians caring for patients with T2DM should continue to focus on smoking cessation, dietary and exercise counseling, BP control, and providing aspirin and statins to a greater extent than achieved even in the ADVANCE and ACCORD studies. For now, rather than changing our current glycemic target, we may best serve our patients with T2DM by implementing programs to help more of them reach the currently recommended goals. ${ }^{29}$ Medical societies such as the ADA or the German Diabetes Association continue to advise most people with T2DM to strive for an $\mathrm{HbA}_{1 \mathrm{c}}$ of $<7 \%$ but it also stresses the need for individualization of treatment goals depending on patient factors including comorbidities (Figure 1). ${ }^{29}$ In view of the recent controversy, the ADA has advised that T2DM patients who have existing CVD or multiple cardiovascular risk factors should consult with their healthcare team about their 'individualized' treatment goals. ${ }^{29}$ On the basis of the data presented, special consideration may now need to be given to high-risk patients with multiple risk factors and heart disease. A target $\mathrm{HbA}_{1 \mathrm{c}}$ level of approximately $7 \%$ may be appropriate in this high-risk population, especially when the use of aggressive pharmacologic therapy is under consideration. As clearly stated by the investigators in the ACCORD trial, the potential existed for undetected adverse effects owing to an increased number of changes in drug regimens and an increased use of multiple drug classes and at increased doses. Thus, all recent studies are important contributions to the field but do not provide a definitive answer to the problem of glycemic control and CVD. If hypoglycemia was indeed a contributing cause of death in the ACCORD trial, future studies of cardiovascular risk reduction should focus on targeting near-normal glycemic levels with the use of strategies and therapies associated with a lower risk of hypoglycemia. Other ongoing clinical trials may provide additional clarification in the near future with this regard. ${ }^{11,35-37}$

\section{Summary}

The contribution of glucose lowering to the reduction of macrovascular events in UKPDS and the ADVANCE and ACCORD trials appears to be minimal, at least in the first few years of treatment. In patients with recently recognized diabetes with no prior CVD events, glycemic control to normal or near-normal levels appears to be effective in preventing CVD events and mortality. In patients with established T2DM ( 8 to 10 or more years) and recognized CVD, however, glycemic control to normal or near-normal levels does not reduce the risk of further CVD events or mortality. Although improved glucose control can clearly protect against the development of microvascular complications, the absence of a reduction in macrovascular events implicates an additive effect of nonglycemic risk factors that often accompany diabetes, such as hypertension, hyperlipidemia 


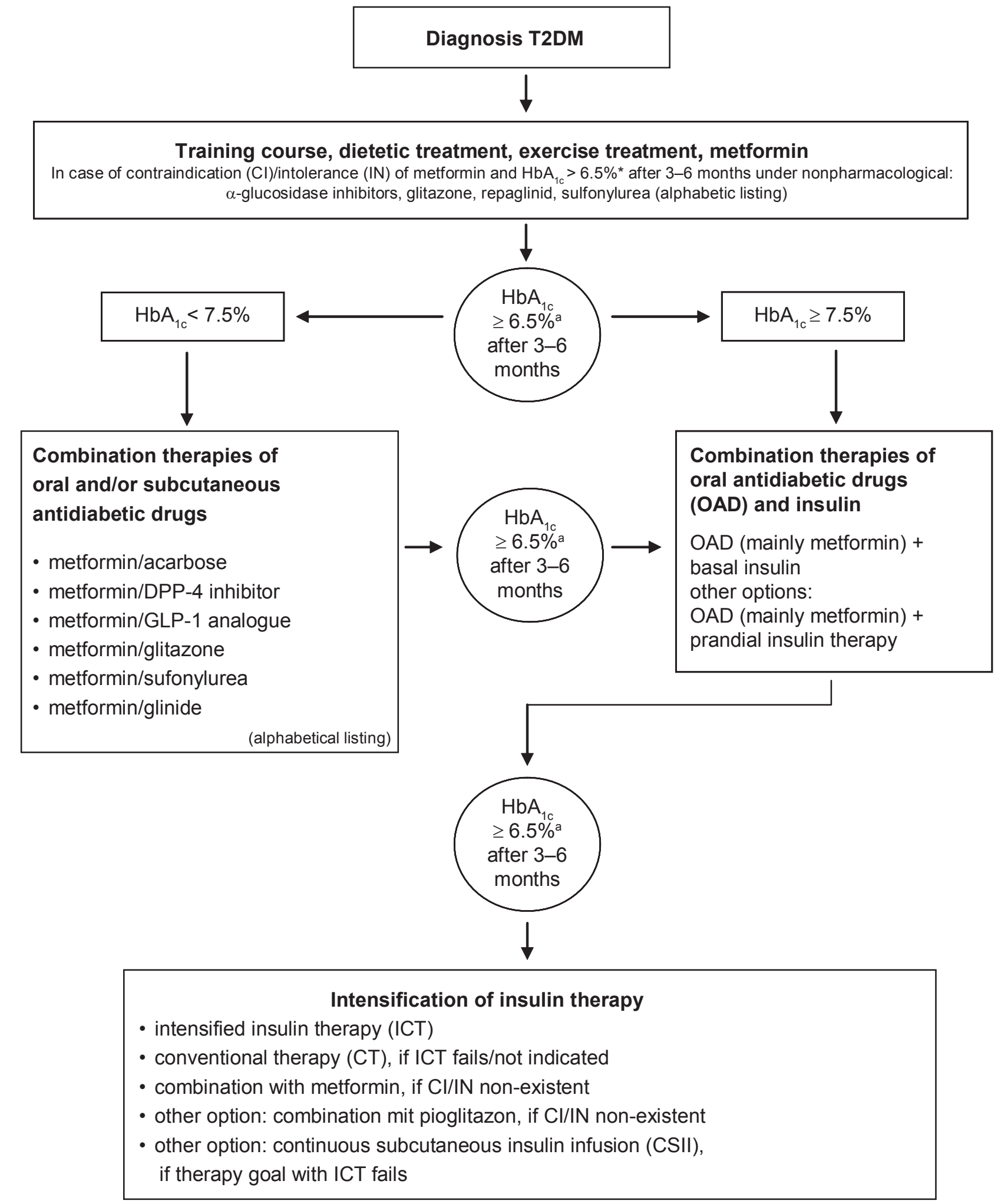

Figure I Evidence-based guideline for antihyperglycemic treatment in patients with T2DM (adopted from the German Diabetes Association [Deutsche Diabetes Gesellschaft/ DDG] based on the data from the ACCORD, ADVANCE, VADT and UKPDS post-trial).

aReduce $\mathrm{HbA}_{\mathrm{Ic}}$ level to $\leq 6.5 \%$ from $\leq 7 \%$ might be advantageous but only when:

- (severe) hypoglycemia is prevented

- weight gain does not occur

- use of multiple glucose-lowering drugs $(>2)$ or additional insulin therapy can be avoided

$\mathrm{HbA}_{\mathrm{lc}}$ should be measured every 3 months. Therapy should be intensified if/when the target level is missed. In contrast, pharmacological dechallenge and 'step back' can be performed if the individual $\mathrm{HbA}_{\mathrm{Ic}}$ remains stable over a longer time. 
and hypercoagulability. Therefore, multifactorial interventions by aggressive management of hypertension, dyslipidemia and hyperglycemia, use of aspirin, and cessation of smoking similar to Steno-2 should be the goal of therapy. There is clear evidence for such an approach where benefits in terms of CVD outcomes remained significant up to a period of 13 years. $^{4}$

\section{Abbreviations}

ACCORD, Action to Control Cardiovascular Risk in Diabetes; ACEI, angiotensin-converting enzyme inhibitor; ADVANCE, Action in Diabetes Vascular Disease-Preterax and Diamicron Modified Release Controlled Evaluation; $\mathrm{ARB}$, angiotensin receptor blocker; $\mathrm{BP}$, blood pressure; CVD, cardiovascular disease; DCCT, Diabetes Control and Complications Trial; $\mathrm{HbA}_{1 \mathrm{c}}$, glycated hemoglobin $\mathrm{A}_{1 \mathrm{c}}$; HOT, Hypertension Optimal Treatment study; LDL, low-density lipoprotein; MI, myocardial infarction; ONTARGET, ONgoing Telmisartan Alone and in combination with Ramipril Global Endpoint Trial; RAS, reninangiotensin system; UKPDS, United Kingdom Prospective Diabetes Study; T1DM, type 1 diabetes mellitus; T2DM, type 2 diabetes mellitus; VADT, Veterans Affairs Diabetes Trial.

\section{Disclosures}

The authors declare that there is no duality of interest associated with this manuscript.

\section{References}

1. Solomon CG. Reducing cardiovascular risk in type 2 diabetes. $N$ Engl J Med. 2003;348(5):457-459.

2. Gaede P, Pedersen O. Target intervention against multiple-risk markers to reduce cardiovascular disease in patients with type 2 diabetes. Ann Med. 2004;36(5):355-366.

3. Gaede P, Vedel P, Larsen N, Jensen GVH, Parving HH, Pedersen O. Multifactorial intervention and cardiovascular disease in patients with type 2 diabetes. N Engl J Med. 2003;348(5):383-393.

4. Gaede P, Lund-Andersen H, Parving HH, Pedersen O. Effect of a multifactorial intervention on mortality in type 2 diabetes. $N$ Engl $J$ Med. 2008;358(6):580-591.

5. Skyler JS, Bergenstal R, Bonow RO, Buse J, Deedwania P, Gale EAM, et al. Intensive Glycemic Control and the Prevention of Cardiovascular Events: Implications of the ACCORD, ADVANCE, and VA Diabetes Trials: A position statement of the American Diabetes Association and a scientific statement of the American College of Cardiology Foundation and the American Heart Association. Diabetes Care. 2009;32(1):187-192.

6. Intensive blood-glucose control with sulphonylureas or insulin compared with conventional treatment and risk of complications in patients with type 2 diabetes (UKPDS 33). UK Prospective Diabetes Study (UKPDS) Group. Lancet. 1998;352(9131):837-853.

7. The Diabetes Control and Complications Trial Research Group. The Effect of intensive treatment of diabetes on the development and progression of long-term complications in insulin-dependent diabetes mellitus. N Engl J Med. 1993;329(14):977-986.
8. The Diabetes Control and Complications Trial/Epidemiology of Diabetes Interventions and Complications (DCCT/EDIC) Study Research Group. Intensive diabetes treatment and cardiovascular disease in patients with type 1 diabetes. N Engl J Med. 2005;353(25):2643-2653.

9. The Action to Control Cardiovascular Risk in Diabetes Study Group. Effects of intensive glucose lowering in type 2 diabetes. $N$ Engl J Med. 2008;358(24):2545-2559.

10. The ADVANCE Collaborative Group. Intensive blood glucose control and vascular outcomes in patients with type 2 diabetes. $N$ Engl J Med. 2008;358(24):2560-2572.

11. Duckworth W, Abraira C, Moritz T, Reda D, Emanuele N, Reaven PD, et al. Glucose control and vascular complications in veterans with type 2 diabetes. N Engl J Med. 2009;360(2):129-139.

12. Holman RR, Paul SK, Bethel MA, Matthews DR, Neil HAW. 10-year follow-up of intensive glucose control in type 2 diabetes. N Engl J Med. 2008;359(15):1577-1589.

13. Cefalu WT. Glycemic targets and cardiovascular disease. $N$ Engl J Med. 2008;358(24):2633-2635.

14. Patel A, MacMahon S, Chalmers J, Neal B, Woodward M, et al. Effects of a fixed combination of perindopril and indapamide on macrovascular and microvascular outcomes in patients with type 2 diabetes mellitus (the ADVANCE trial): a randomised controlled trial. Lancet. 2007;370(9590):829-840.

15. Karalliedde J, Gnudi L. ACCORD and ADVANCE: a tale of two studies on the merits of glycaemic control in type 2 diabetic patients. Nephrol Dial Transplant. 2008;23(6):1796-1798.

16. Chalmers J, Cooper ME. UKPDS and the legacy effect. N Engl J Med. 2008;359(15):1618-1620.

17. 2003 European Society of Hypertension-European Society of Cardiology guidelines for the management of arterial hypertension. J Hypertens. 2003;21(6):1011-1053.

18. Ruilope L. New ADVANCEs in guidelines. J Hypertens Suppl. 2008;26(2):S16-S18.

19. Mogensen C. New treatment guidelines for a patient with diabetes and hypertension. J Hypertens Suppl. 2003;21(1):S25-S30.

20. Abuissa H, Jones PG, Marso SP, O'Keefe JH Jr. Angiotensin-converting enzyme inhibitors or angiotensin receptor blockers for prevention of type 2 diabetes: A meta-analysis of randomized clinical trials. $J \mathrm{Am}$ Coll Cardiol. 2005;46(5):821-826.

21. The ONTARGET Investigators. Telmisartan, ramipril, or both in patients at high risk for vascular events. $N$ Engl J Med. 2008;358(15):1547-1559.

22. Davis BR, Furberg CD, Wright JT Jr, Cutler JA, Whelton P; the ALLHAT Collaborative Research Group. ALLHAT: Setting the record straight. Ann Intern Med. 2004;141(1):39-46.

23. Salvetti A, Ghiadoni L. Thiazide diuretics in the treatment of hypertension: an update. J Am Soc Nephrol. 2006;17(4 Suppl 2):S25-S29.

24. D'Agostino RB, Belanger AJ, Kannel WB, Cruickshank JM. Relation of low diastolic blood pressure to coronary heart disease death in presence of myocardial infarction: the Framingham Study. BMJ. 1991;303(6799):385-389.

25. Stamler J, Neaton JD. The Multiple Risk Factor Intervention Trial (MRFIT) - importance then and now. JAMA. 2008;300(11):1343-1345.

26. UK Prospective Diabetes Study Group. Tight blood pressure control and risk of macrovascular and microvascular complications in type 2 diabetes: UKPDS 38. BMJ. 1998;317(7160):703-713.

27. Ruilope L, Hansson L, Zanchetti A. What have we learned from the HOT (hypertension optimal treatment) study? Nephrol Dial Transplant. 1997;12(10):2047-2048.

28. Holman RR, Paul SK, Bethel MA, Neil HAW, Matthews DR. Long-term follow-up after tight control of blood pressure in type 2 diabetes. $\mathrm{NEngl}$ J Med. 2008;359(15):1565-1576.

29. American Diabetes Association. Standards of medical care in diabetes - 2009. Diabetes Care. 2009;32(Suppl 1):S13-S61.

30. Buse JB, Ginsberg HN, Bakris GL, Clark NG, Costa F, Eckel R, et al. Primary prevention of cardiovascular diseases in people with diabetes mellitus: A scientific statement from the American Heart Association and the American Diabetes Association. Diabetes Care. 2007;30(1):162-172. 
31. Bolen S, Feldman L, Vassy J, Wilson L, Yeh H-C, Marinopoulos S, et al. Systematic review: comparative effectiveness and safety of oral medications for type 2 diabetes mellitus. Ann Intern Med. 2007;147(6): 386-399.

32. Krentz A. Comparative safety of newer oral antidiabetic drugs. Expert Opin Drug Saf. 2006;5(6):827-834.

33. Krumholz HM, Lee TH. Redefining quality - implications of recent clinical trials. $N$ Engl J Med. 2008;358(24):2537-2539.

34. Stratton IM, Adler AI, Neil HAW, Matthews DR, Manley SE, Cull CA, et al. Association of glycaemia with macrovascular and microvascular complications of type 2 diabetes (UKPDS 35): prospective observational study. BMJ. 2000;321(7258):405-412.
35. Milicevic Z, Raz I, Strojek K, Skrha J, Tan M, Wyatt J, et al. Hyperglycemia and its effect after acute myocardial infarction on cardiovascular outcomes in patients with Type 2 diabetes mellitus (HEART2D) Study design. J Diabetes Complications. 2005;19(2):80-87.

36. Gerstein H, Yusuf S, Riddle M, Ryden L, Bosch J. Rationale, design, and baseline, Origin Trial Investigators, characteristics for a large international trial of cardiovascular disease prevention in people with dysglycemia: the ORIGIN Trial (Outcome Reduction with an Initial Glargine Intervention). Am Heart J. 2008;155(1):26-32, 32.e1-e6.

37. Magee M, Isley W. Rationale, design, and methods for glycemic control in the Bypass Angioplasty Revascularization Investigation 2 Diabetes (BARI 2D) Trial. Am J Cardiol. 2006;97(12A):20G-30G.
Vascular Health and Risk Management

\section{Publish your work in this journal}

Vascular Health and Risk Management is an international, peerreviewed journal of therapeutics and risk management, focusing on concise rapid reporting of clinical studies on the processes involved in the maintenance of vascular health; the monitoring, prevention and treatment of vascular disease and its sequelae; and the involvement of

\section{Dovepress}

metabolic disorders, particularly diabetes. This journal is indexed on PubMed Central and MedLine. The manuscript management system is completely online and includes a very quick and fair peer-review system, which is all easy to use. Visit http://www.dovepress.com/ testimonials.php to read real quotes from published authors.

Submit your manuscript here: http://www.dovepress.com/vascular-health-and-risk-management-journal 